\title{
ESTUDO HIDROLÓGICO E MODELAGEM CHUVA-VAZÃO DA BACIA DO RIO DOCE
} Arthur da Fontoura Tschiedel ${ }^{1}$ Bibiana Rodrigues Colossi ${ }^{2}$

Resumo: Este artigo se resume ao estudo hidrológico da bacia do Rio Doce. Na cabeceira desta bacia ocorreu o rompimento de uma barragem de rejeitos que resultou em perdas humanas e num grande desastre ambiental. E, devido a este fato recente, este trabalho procurou subsidiar, a partir de simulação hidrológica, estudos posteriores de rompimentos de barragens na região, que necessitem de dados de vazão e velocidade aproximada de escoamento em trechos discretizados ao longo do Rio Doce. O MGB-IPH foi calibrado com a utilização de 18 estações fluviométricas espalhadas ao longo da bacia, 431 estações pluviométricas e 42 estações com dados de clima. Os coeficientes de eficiência utilizados para avaliar a relação entre as vazões calculadas e as vazões observadas variaram de 0.334 a 0.648 para as 18 sub-bacias, considerando altas vazões, e de 0.158 a 0.744 considerando períodos de baixas vazões. No exutório considerado para a bacia, o ajuste ficou em 0.533 e 0.700 para o NS e o LOGNS, respectivamente. Espera-se, portanto, com este trabalho, fornecer subsídios para posteriores trabalhos envolvendo o estudo do deslocamento da onda de lama ao longo do Rio Doce para diferentes momentos da curva de permanência.

Abstract: This article summarizes the hydrological study of the basin of the Rio Doce. At the head of this basin was the rupture of a dam which resulted in loss of life and a major environmental disaster. And because of this recent event, this study aims, support from hydrologic simulation, further studies of dam breaks in the region, requiring flow data and approximate speed of flow in discretized reaches along the Rio Doce. The MGB-IPH model was calibrated using 18 gauged stations located along the basin, 431 rainfall stations and 42 stations with climate data. Efficiency coefficients used to evaluate the relationship between the calculated flow and the observed flow rates ranged from 0.334 to 0.648 for the 18 sub-basins, considering high flow rates, and 0.158-0.744 considering periods of low flow. In downstream point considered for the basin, the adjustment was in 0.533 and 0.700 for the NS and LOGNS respectively. It is expected, therefore, with this work, provide support for further work involving the study of the progress of the wave of mud along the Rio Doce for different times of the duration curve.

Palavras-Chave: Rio Doce; Modelagem Chuva-Vazão; MGB-IPH

\footnotetext{
${ }^{1}$ Instituto de Pesquisas Hidráulicas - UFRGS. Avenida Bento Gonçalves, 9500. Porto Alegre, RS. Fone: (51) 3308-6670

E-mail: arthurtidel@hotmail.com

${ }^{2}$ Instituto de Pesquisas Hidráulicas - UFRGS. Avenida Bento Gonçalves, 9500. Porto Alegre, RS. Fone: (51) 3308-6670

E-mail: bibiana.colossi@gmail.com
} 


\section{1 - INTRODUÇÃO}

O desenvolvimento tecnológico da sociedade está associado a diversos riscos, em que, no contexto deste trabalho, são focadas as tecnologias que concernem o desenvolvimento e implantação de barragens de rejeito ao longo do Brasil, e os impactos ambientais decorrentes das mesmas. Barragens de rejeito são estruturas de terra construídas para armazenar resíduos de mineração, os quais são definidos como a fração estéril produzida pelo beneficiamento de minérios, seja em processo mecânico ou químico. O rejeito é um material que não possui valor econômico e que deve ser devidamente armazenado. De acordo com Passos (2009), os rejeitos de mineração podem ter três etapas de comportamento: (i) Comportamento de lâmina líquida; (ii) Comportamento semi-líquido e semi-viscoso e; (iii) Comportamento de solo.

Um dos grandes riscos associados às barragens de rejeitos é referente ao rompimento das mesmas, e este é um dos aspectos considerados neste trabalho: O comportamento hidrológico de uma bacia cuja cabeceira é utilizada como local para construção de barragens de rejeitos deve ser muito bem estudado para que possa subsidiar a elaboração de planos de ação de emergências em possíveis momentos distintos de rompimento de barragens. Neste sentido comparar-se-á os resultados de monitoramentos de vazões no rio principal de uma bacia onde ocorreu um desastre relacionado ao rompimento de barragens de rejeitos com resultados advindos de técnicas de modelagem hidrológica. Assim, espera-se contribuir para o entendimento hidrológico da bacia, além de fornecer subsídios para que posteriormente possam ser simulados deslocamentos de picos de cheia decorrentes de rompimento de barragens em diferentes épocas de ano.

A bacia foco de estudo onde ocorreu o desastre ambiental é a bacia do Rio Doce localizada em MG e ES. O acidente ocorrido no dia 05/11/2015, decorrente do rompimento da barragem de rejeitos Fundão, da empresa Samarco, localizada no município de Mariana (MG) resultou em aproximadamente $34 \mathrm{hm}^{3}$ de rejeitos da mineração de ferro lançados na bacia do Rio Doce. $\mathrm{O}$ evento foi responsável pela perda de muitas vidas humanas e também pela degradação de grande parte da bacia do Rio Doce, de acordo com IBAMA (2015). A seguir é apresentado um mosaico de fotos da região para que se possa ter uma ideia da magnitude do impacto no local do acidente e do impacto no encontro da onda de detritos com o mar (Figura 1). 

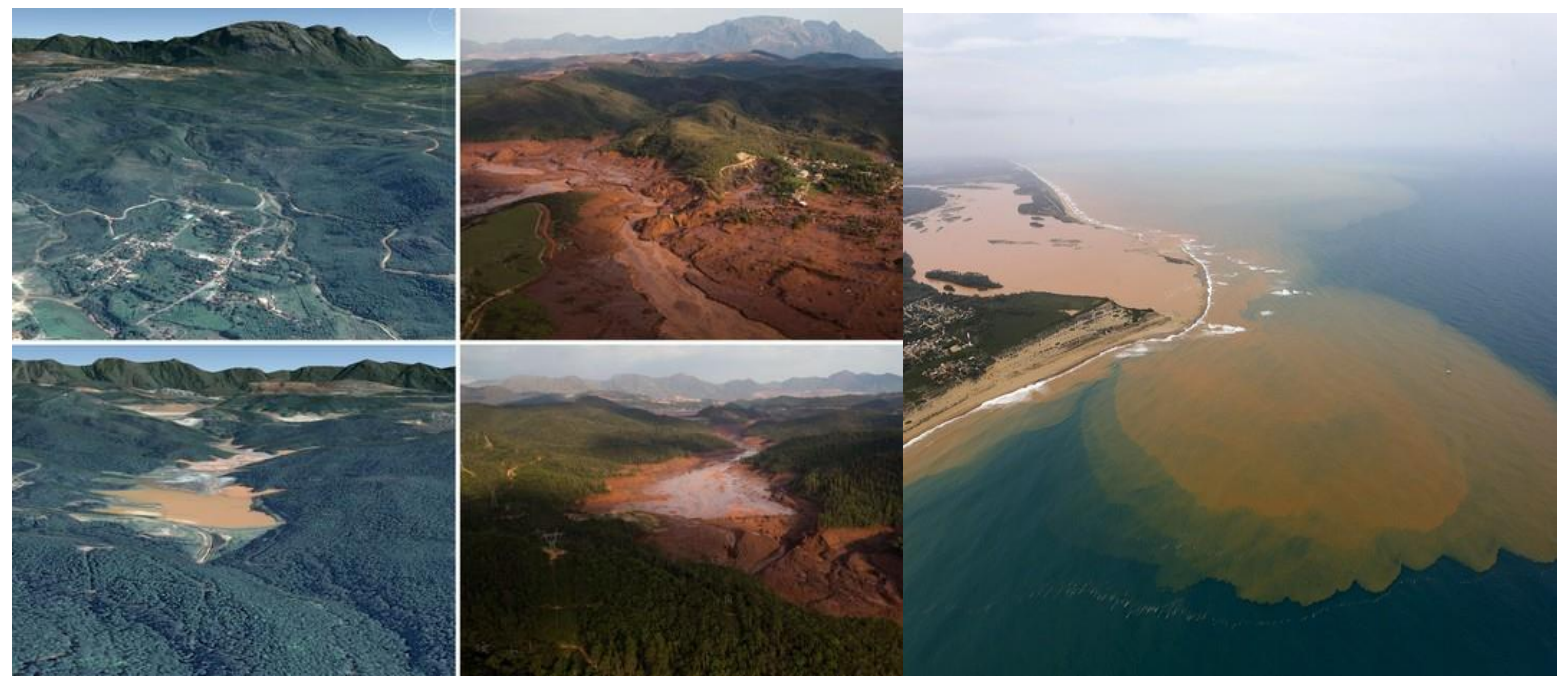

Figura 1 - Antes e depois no local do acidente

O deslocamento do pico de cheia e do pico de concentração de sedimentos ao longo do Rio Doce foi monitorado em algumas localidades pela CPRM, de modo que no laudo técnico preliminar elaborado pelo IBAMA (2015), aponta que o primeiro contato da onda de lama com o mar foi no dia 21/11/2015. Ou seja, foram necessários 16 dias para que a onda de lama percorresse todo o Rio Doce (Figura 2) no começo do mês de novembro, que é um período de vazões médias na bacia (Figura 3).

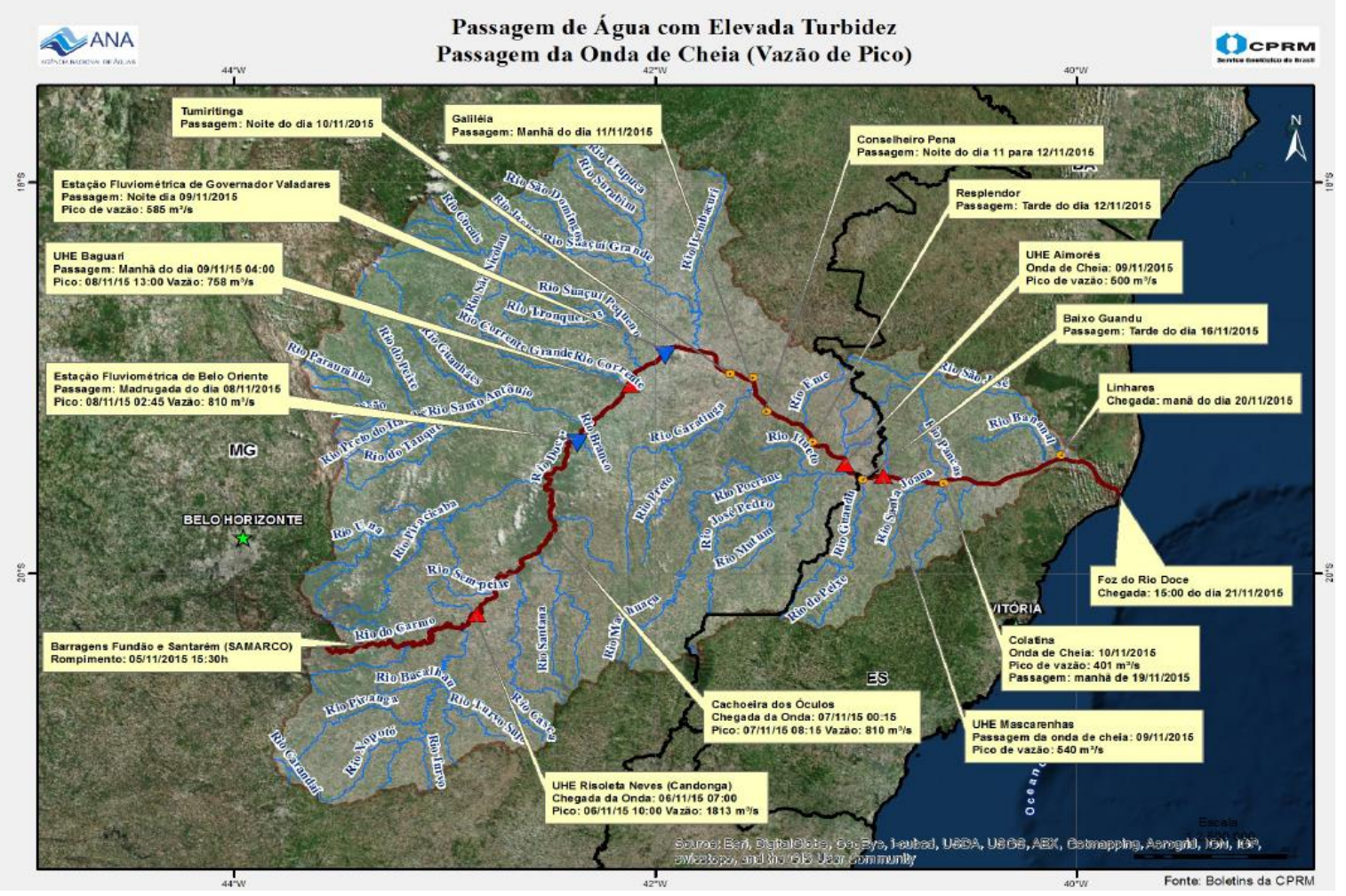

Figura 2-Caminho da onda de lama ao longo do Rio Doce

Por fim, o modelo utilizado para gerar dados de vazão sintéticos que possam posteriormente servir como embasamento para simular o deslocamento da onda de lama decorrente de rompimento de barragens em diferentes valores da curva de permanência, foi o Modelo de Grandes Bacias MGB- 
IPH (Collischonn et al., 2007), que forneceu dados de vazão e velocidade para diferentes momentos da curva de permanência do Rio Doce.

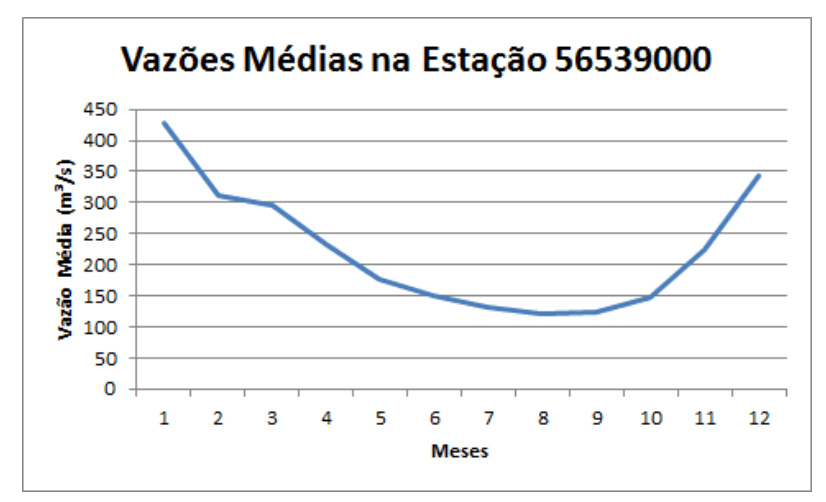

Figura 3-Vazões Médias Mensais na Estação 56539000 - Colatina

\section{2 - METODOLOGIA}

A metodologia do estudo consistiu em buscar dados de entrada e calibrar a bacia do Rio Doce no MGB-IPH a partir de dados de vazão, chuva e clima monitorados pelas estações presentes na bacia (ou proximidades, no caso das Estações Pluviométricas), para um exutório considerado na Estação Fluviométrica 56994500, localizada no Espírito Santo, no município de Colatina. No processo de calibração, ainda, dividiu-se a Bacia do Rio Doce em 18 sub-bacias com diferentes características de tipo de solo e uso do solo, na tentativa de representar a heterogeneidade da bacia.

\section{1 - Descrição Geral do Modelo MGB-IPH}

O modelo MGB-IPH é um modelo do tipo chuva-vazão distribuído focado para grandes bacias hidrográficas (Collischonn et al., 2007) que utiliza, como dados de entrada, diversas informações relacionadas ao ciclo hidrológico e às características físicas da bacia em estudo, assim como grande parte dos modelos hidrológicos distribuídos desenvolvidos nos últimos anos (Tucci, 2005). Desse modo, informações relativas à chuva, vazão, evapotranspiração e outros dados climáticos medidos em postos pluviométricos e fluviométricos (ou estimados por outros métodos não aqui abordados) fazem parte dos dados de entrada no modelo. Informações relativas à altimetria, direções de fluxos, rede de drenagem, uso e ocupação da bacia e tipo de solo também são dados de entrada, bem como outros parâmetros como altura média da vegetação, índice de área foliar, albedo e resistência da superfície para condições de umidade do solo.

O modelo trabalha a partir da discretização de minibacias considerando a rede de drenagem obtida a partir do Modelo Digital de Elevação (MDE) utilizado. Para a simulação hidrológica, essas minibacias que compõe a bacia hidrográfica são divididas em Unidades de Respostas Hidrológicas (URHs), que é uma combinação entre o uso do solo e o tipo de solo para uma dada bacia, representando a heterogeneidade da bacia em relação à geração de escoamento. Desse modo, para 
cada minibacia considerada são calculadas as porcentagens de cada URH, que estão diretamente relacionadas à como o escoamento será gerado neste local. O modelo permite também escolher dois métodos para representar a propagação do escoamento: O método Muskingun-Cunge (considerado como método de propagação de escoamento no âmbito deste trabalho) e o Método Inercial. A estruturação geral do modelo é apresentada a seguir (Figura 4).

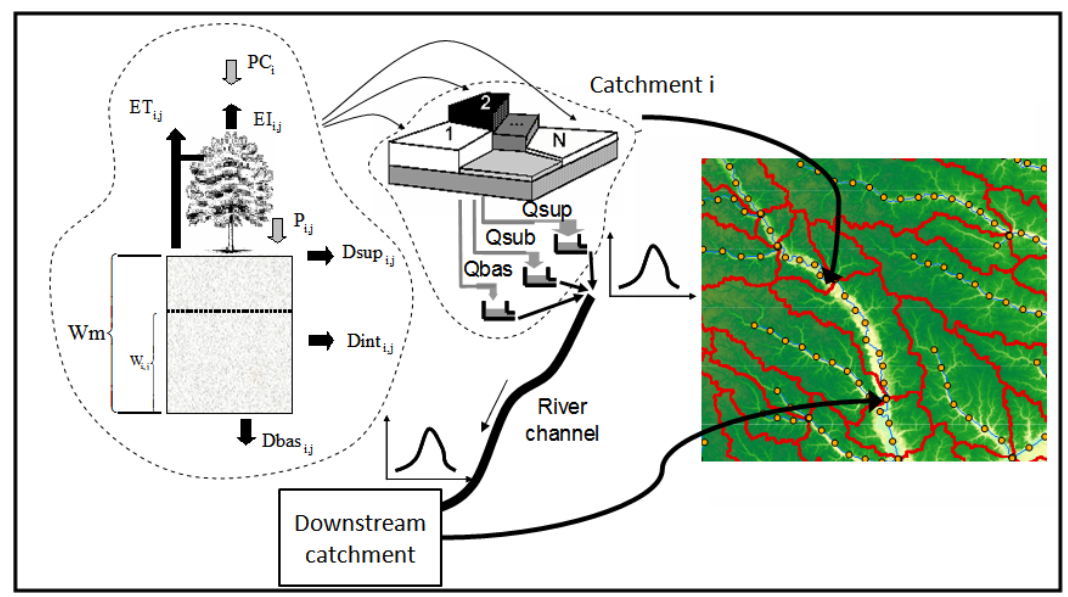

Figura 4-Descrição geral do Modelo MGB-IPH

O módulo responsável por gerar escoamento em cada mini-bacia discretizada possui 11 parâmetros calibráveis por sub-bacia considerada. Estes parâmetros, em conjunto com o significado e faixas de variação, são apresentados na Tabela 1.

Tabela 1 - Parâmetros Calibráveis do MGB e Significados

\begin{tabular}{|l|l|l|}
\hline Parâmetro & Valores & Significado \\
\hline WM & $50-1000$ & Capacidade de Armazenamento do Solo \\
\hline b & $0.12-1.6$ & Correlação entre o armazenamento e saturação do solo \\
\hline Kbas & $0.05-5$ & Controla a vazão durante a estiagem \\
\hline Kint & $4-40$ & Controla a quantidade de água na camada do solo \\
\hline XL & 0.67 & Controla o perfil da redução da curva de água na superfície \\
\hline CAP & 0 & Controla a vazão do reservatório de subsolo \\
\hline WC & 0.1 & Parâmetro de armazenamentos residuais \\
\hline CB & $1200-8000$ & Parâmetro do reservatório linear simples \\
\hline QB & 0.1 & Vazão de base do início da simulação \\
\hline CS & $1-20$ & Parâmetro de calibração do espalhamento da superfície \\
\hline CI & $50-200$ & Parâmetro de calibração do espalhamento da sub-superfície \\
\hline
\end{tabular}

A calibração do modelo é realizada com base na observação entre as vazões calculadas para a bacia e as vazões observadas em postos fluviométricos, de modo que os resultados provenientes de uma simulação são avaliados a partir do coeficiente de eficiência Nash e Sutcliffe $\left(\mathrm{R}^{2}\right)$, que é um dos 
parâmetros frequentemente utilizados na avaliação do desempenho de modelos hidrológicos com maior sensibilidade para altas vazões (Equação 01). Este coeficiente pode variar do negativo infinito até 1, quando seu ajuste é perfeito. Ainda, o desempenho de um modelo hidrológico é considerado adequado se $\mathrm{R}^{2}$ é superior a 0,75 e aceitável se o valor de $\mathrm{R}$ fica entre 0,36 e 0,75 (Silva et al., 2008). Também foi utilizado o R adaptado para o logaritmo das vazões, que dá mais peso para as vazões de recessão do hidrograma (Equação 02) e o erro relativo de volume total dos hidrogramas (Equação 03). As equações relativas aos coeficientes de eficiência do modelo são apresentadas a seguir.

Coeficiente ENS:

$$
E_{N S}=1-\frac{\sum\left(Q_{t}^{o b s}-Q_{t}^{c a l c}\right)^{2}}{\sum\left(Q_{t}^{o b s}-\overline{Q_{t}^{o b s}}\right)^{2}}
$$

Coeficiente ENSlog:

$$
E_{N S l o g}=1-\frac{\sum\left(\log Q_{t}^{o b s}-\log Q_{t}^{c a l c}\right)^{2}}{\sum\left(\log Q_{t}^{o b s}-\log \overline{Q_{t}^{o b s}}\right)^{2}}
$$

Coeficiente Erro de Volume:

$$
\Delta \mathrm{V}=\frac{\sum Q_{t}^{\text {calc }}-\sum Q_{t}^{o b s}}{\sum Q_{t}^{o b s}}
$$

Em que $\mathrm{Q}_{\mathrm{t}}{ }^{\mathrm{obs}}$ são as vazões observadas no tempo t; $\mathrm{Q}_{\mathrm{t}}{ }^{\text {calc }}$ são as vazões calculadas no tempo t e; $\overline{\mathrm{Q}_{\mathrm{t}}^{\text {obs }}}$ é a média das vazões observadas.

\section{2 - Dados de Entrada Utilizados}

Como dados de entrada climáticos no modelo MGB-IPH, foram inseridas 42 estações pluviométricas com dados de clima, 18 estações fluviométricas com dados de vazões e 431 estações pluviométricas com dados de chuva. A Figura 5 mostra a localização das estações pluviométricas e fluviométricas utilizadas no estudo, bem como a localização da onde ocorreu o acidente em relação ao Rio Doce.

Como dados de entrada relacionados à parte física da bacia, foram utilizados: (i) o Modelo Digital de Elevação SRTM, com 90 metros de resolução espacial; (ii) Shape de Uso dos Solos do Brasil, fornecido pelo IBGE e; (iii) Shape de solo para o Brasil, também fornecido pelo IBGE. Já para os dados relacionados aos chamados parâmetros fixos do modelo, que são a altura média da vegetação, o índice de área foliar, o albedo e resistência da superfície para condições de umidade do solo foram considerados os valores indicados pelo próprio MGB. 


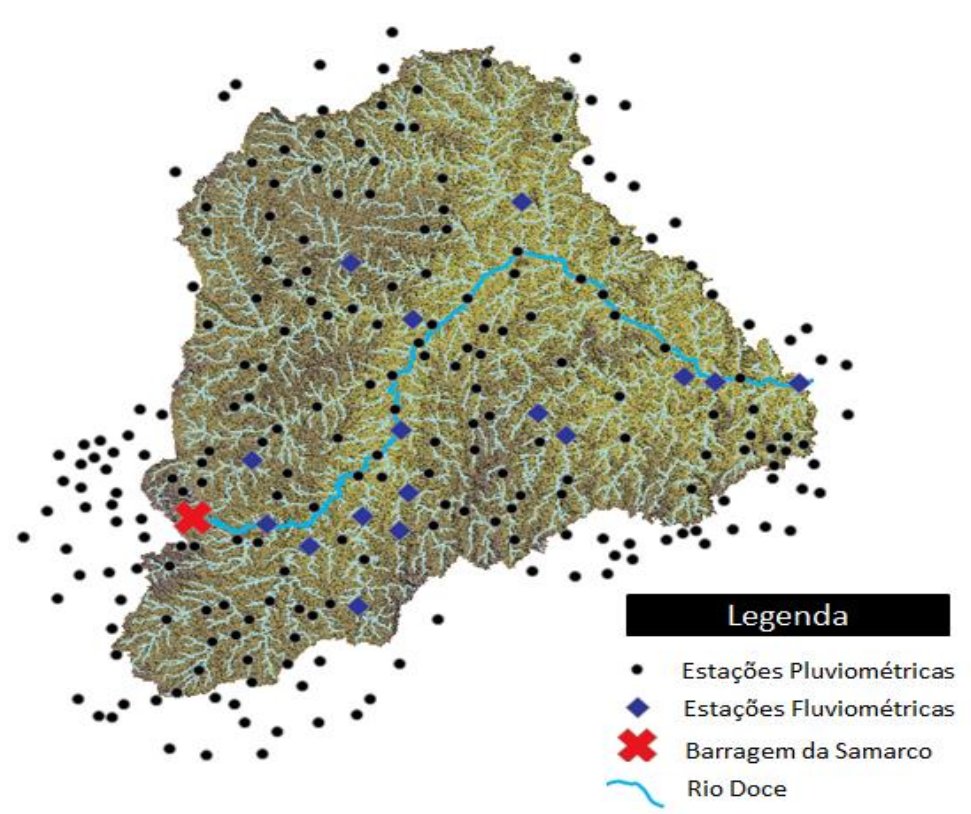

Figura 5-Dados de Entrada de Chuva e Vazão Inseridos no MGB

O período de simulação utilizado foi de 1970 a 2014, o que corresponde a 44 anos de dados observados, destacando-se que este extenso período utilizado acarreta certas dificuldades para a realização da calibração do modelo, uma vez que, durante o período, mudanças de uso de solo significativas podem ter ocorrido, o que pode incluir também a construção de reservatórios e consequentes regularizações das vazões observadas. A seguir (Figura 6) é apresentada a disponibilidade de dados de vazão observados para o período, a partir de um gráfico de Gannt.

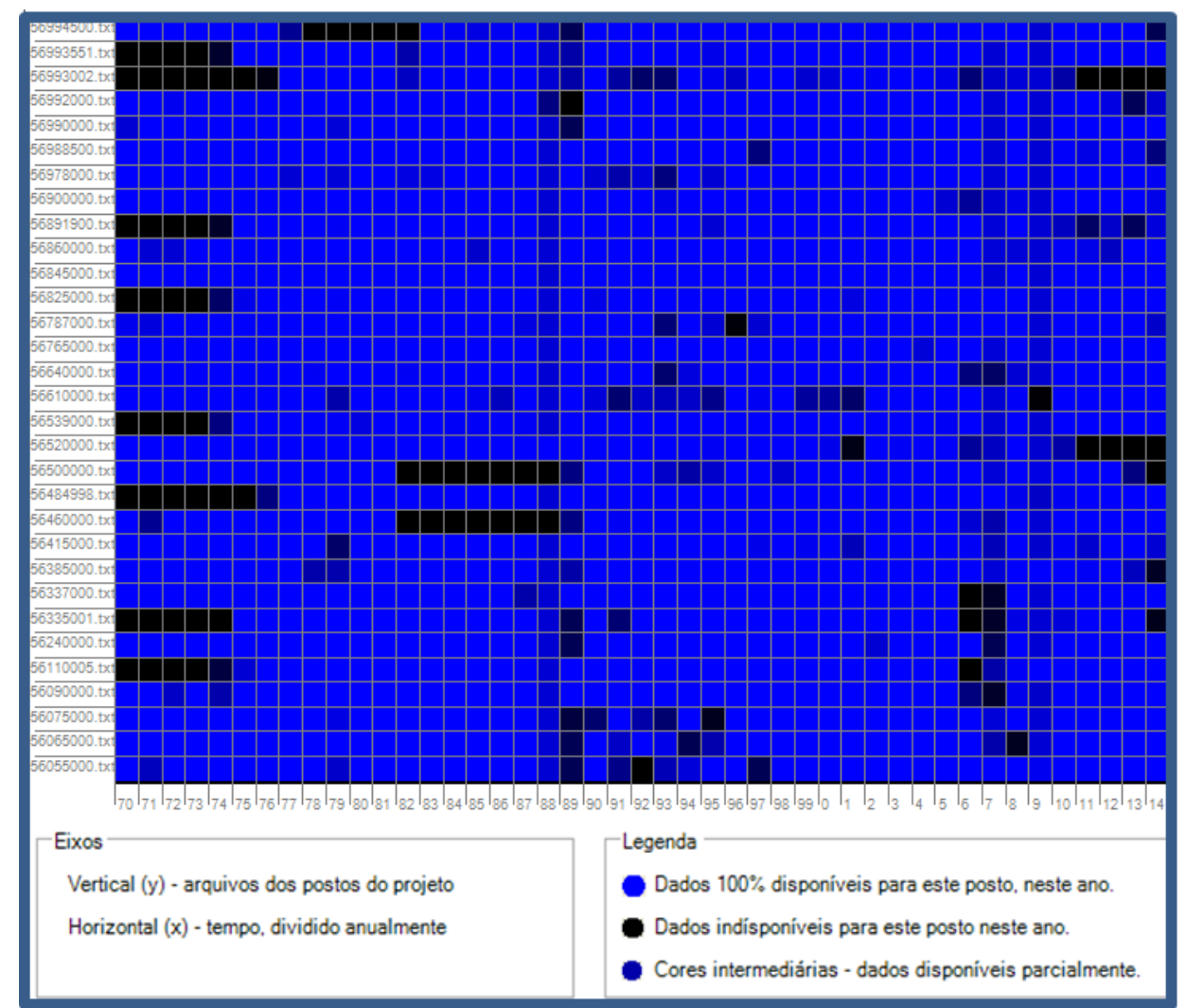

Figura 6-Disponibilidade temporal dos dados de vazão utilizados para a calibração 


\section{3 - RESULTADOS}

O processo de simulação da bacia do Rio Doce no MGB-IPH considerou 8 URHs, em que majoritariamente observou-se que as URHs de Pastagem em Solo Profundo e Floresta em Solo Profundo dominam a bacia (Figura 7). Ainda, na Figura 8 são apresentadas as sub-bacias utilizadas no processo de calibração do modelo.

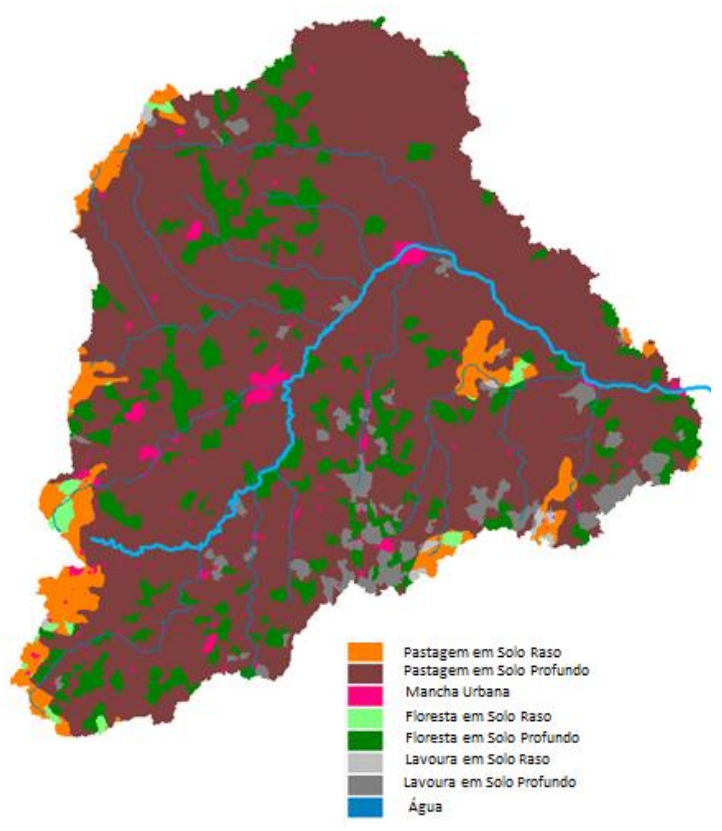

Figura 7-Unidades de Respostas Hidrológicas consideradas no estudo

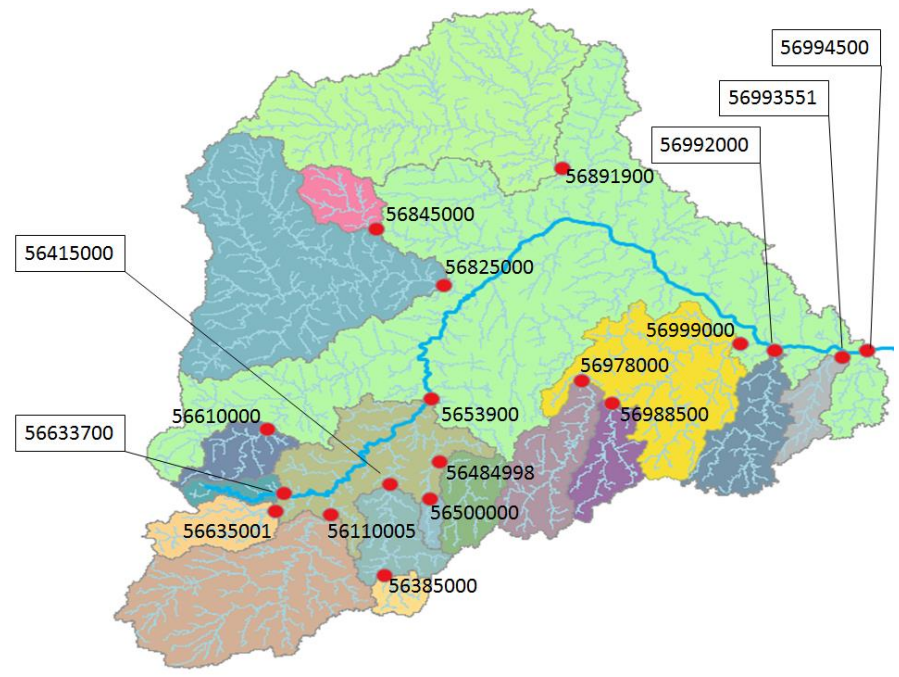

Figura 8-Sub-Bacias consideradas no estudo

O processo de calibração considerou 18 sub-bacias, gerando, portanto 18 comparações entre dados calculados e dados observados, para diferentes porções da bacia e 18 conjuntos de parâmetros calibráveis. Dentre todos os NS calculados, destaca-se que em alguns o ajuste não pode ser considerado como aceitável segundo Silva et al. (2008). A seguir (Figura 9) são apresentados alguns dos melhores resultados obtidos. 


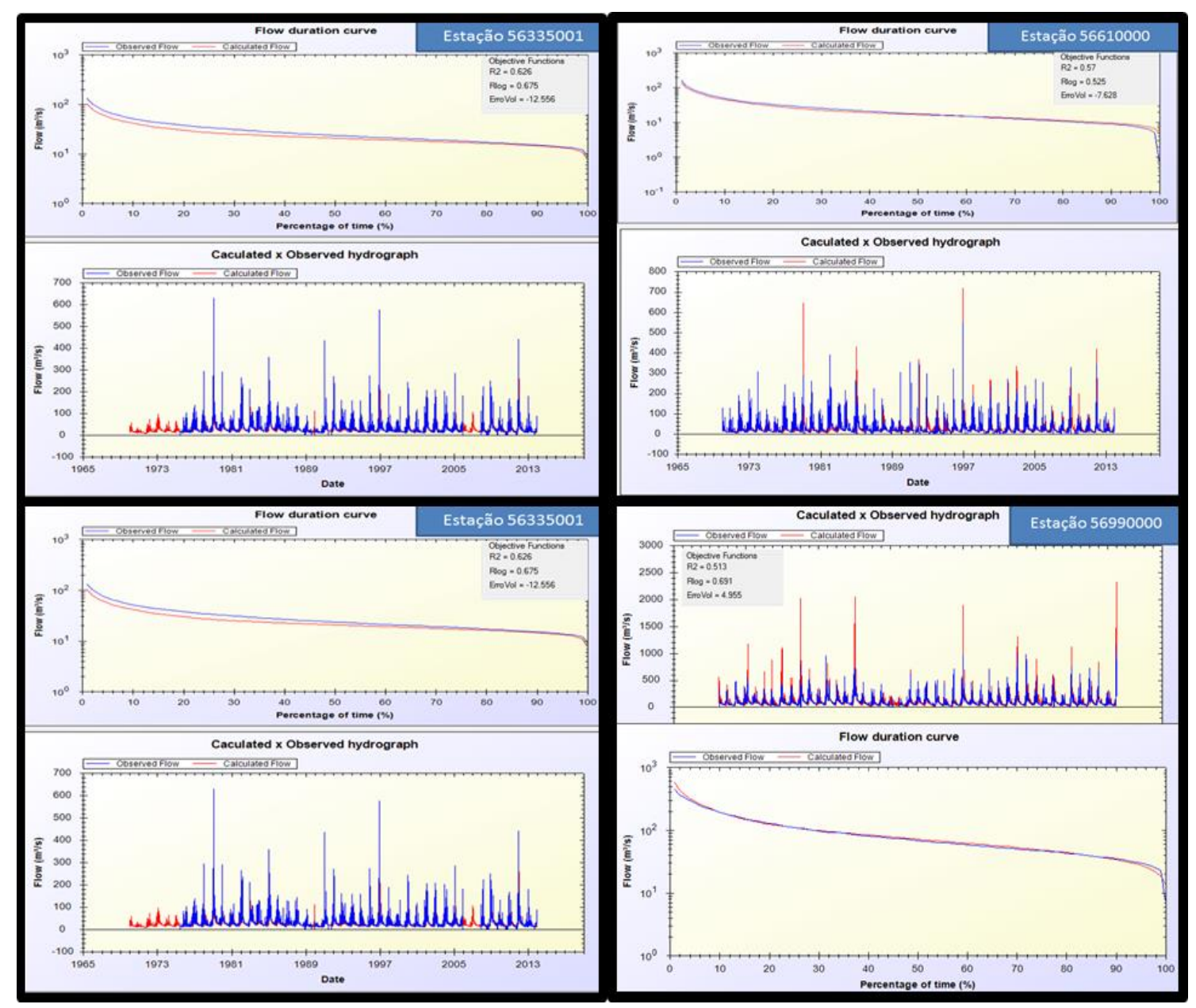

Figura 9-Melhores resultados das calibrações

Na Figura 10 e na Figura 11 são apresentados os resultados obtidos no exutório da bacia, para todo o período simulado e para um período aleatoriamente escolhido entre 2003 e 2007, que teve a finalidade de apresentar de forma mais ampliada os resultados alcançados. Ainda, na Tabela 2 é apresentado um resumo com todos os coeficientes de eficiência obtidos para as sub-bacias. Ainda, no Anexo estão todos os parâmetros utilizados na calibração para cada uma das sub-bacias.

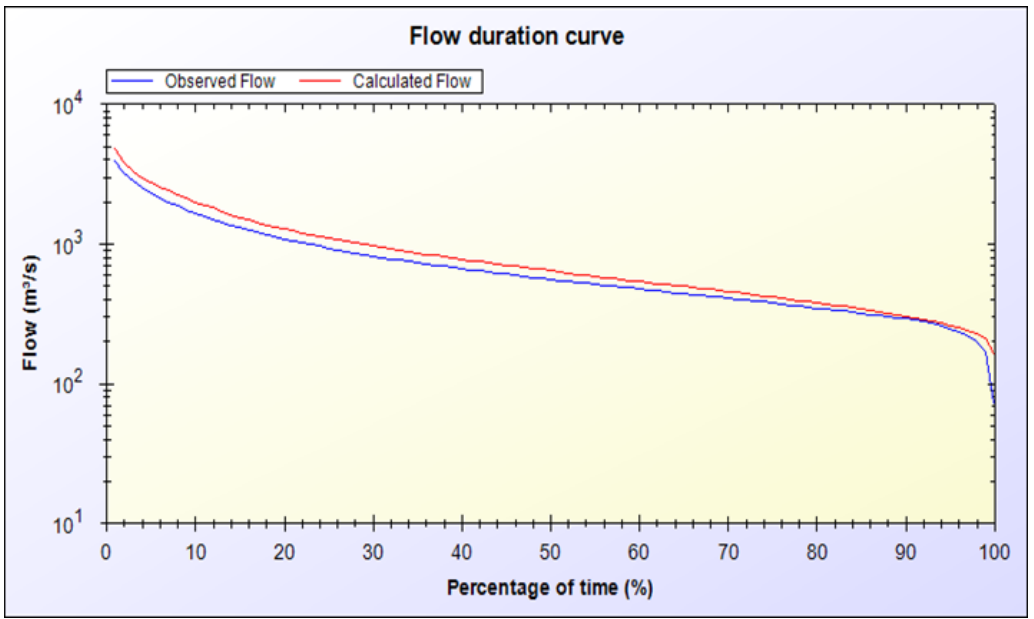

Figura 10-Curva de Permanência Calculada e Observada na Estação 56539000 


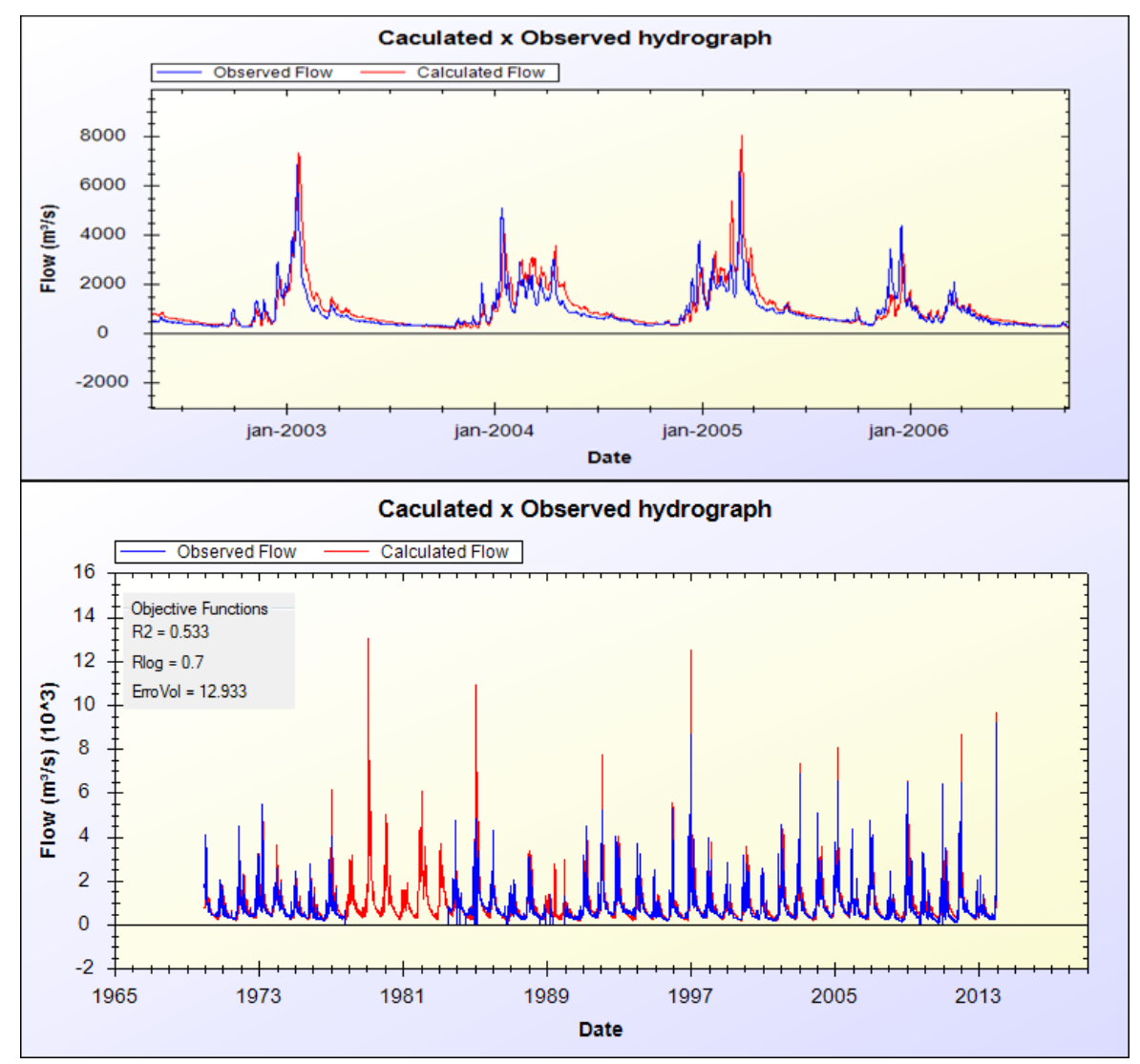

Figura 11-Resultado no exutório considerado (Estação 56994500)

Tabela 2 -Coeficientes de Eficiência por Sub-Bacia

\begin{tabular}{|c|c|c|c|c|c|}
\hline $\begin{array}{l}\text { Sub- } \\
\text { Bacia }\end{array}$ & Código Estação & $\mathrm{R} 2$ & Rlog & ErroVol & Área de Drenagem $\left(\mathrm{km}^{2}\right)$ \\
\hline 1 & 56994500 & 0.533 & \begin{tabular}{|l|}
0.700 \\
\end{tabular} & 12.933 & 76400 \\
\hline 2 & 56993551 & 0.588 & 0.539 & -5.767 & 893 \\
\hline 3 & 56992000 & 0.469 & \begin{tabular}{|l|}
0.467 \\
\end{tabular} & -4.463 & 2130 \\
\hline 4 & 56990000 & 0.513 & 0.691 & 4.955 & 8720 \\
\hline 5 & 56978000 & 0.439 & 0.475 & -13.804 & 2350 \\
\hline 6 & 56988500 & 0.648 & 0.688 & -0.799 & 1410 \\
\hline 7 & 56539000 & 0.517 & 0.588 & 15.86 & 15900 \\
\hline 8 & 56337000 & 0.542 & 0.744 & -2.673 & 529 \\
\hline 9 & 56484998 & 0.517 & 0.42 & 0.154 & 1350 \\
\hline 10 & 56500000 & 0.464 & 0.158 & -13.604 & 273 \\
\hline 11 & 56415000 & 0.334 & 0.205 & -3.858 & 2030 \\
\hline 12 & 56110005 & 0.577 & 0.528 & 9.555 & 6230 \\
\hline 13 & 56335001 & 0.626 & 0.675 & -12.556 & 1370 \\
\hline 14 & 56610000 & 0.57 & 0.525 & -7.628 & 1160 \\
\hline 15 & 56825000 & 0.637 & 0.634 & -18.37 & 10200 \\
\hline 16 & 56845000 & $\begin{array}{c}- \\
1.292\end{array}$ & 0.428 & 33.068 & 1050 \\
\hline 17 & 56891900 & 0.349 & 0.627 & 14.596 & 9770 \\
\hline 18 & 56385000 & 0.445 & 0.559 & -12.316 & 523 \\
\hline
\end{tabular}




\section{4 - CONCLUSÕES}

Avalia-se que os resultados dos coeficientes de eficiência utilizados se demonstraram, para a maior parte dos casos, aceitável (de acordo com a faixa de valores proposta por Silva et al., 2008). Entretanto, de acordo com esta metodologia nenhum coeficiente de eficiência pode ser considerado como "adequado" para a bacia. A visualização dos resultados é feita a partir da Figura 12, em que o coeficiente de $\mathrm{R}^{2}$ (para altas vazões) variou da ordem de 0.334 a 0.648 , e o coeficiente RLOG (para baixas vazões) variou de 0.158 a 0.744 . Ressalta-se que nesta figura excluiu-se o $\mathrm{R}^{2}$ de $-1,292$ obtido para a sub-bacia 16. Esta exclusão foi feita uma vez que a análise entre a vazão calculada e a vazão observada para um único evento no ano de 1971 desta sub-bacia leva a acreditar que existem problemas relativos aos dados de entrada de chuva para a bacia, neste período. Outra hipótese são erros na leitura das vazões observadas para esta data, considerando a enorme diferença de magnitude entre os dados observados e os dados calculados só para um evento.

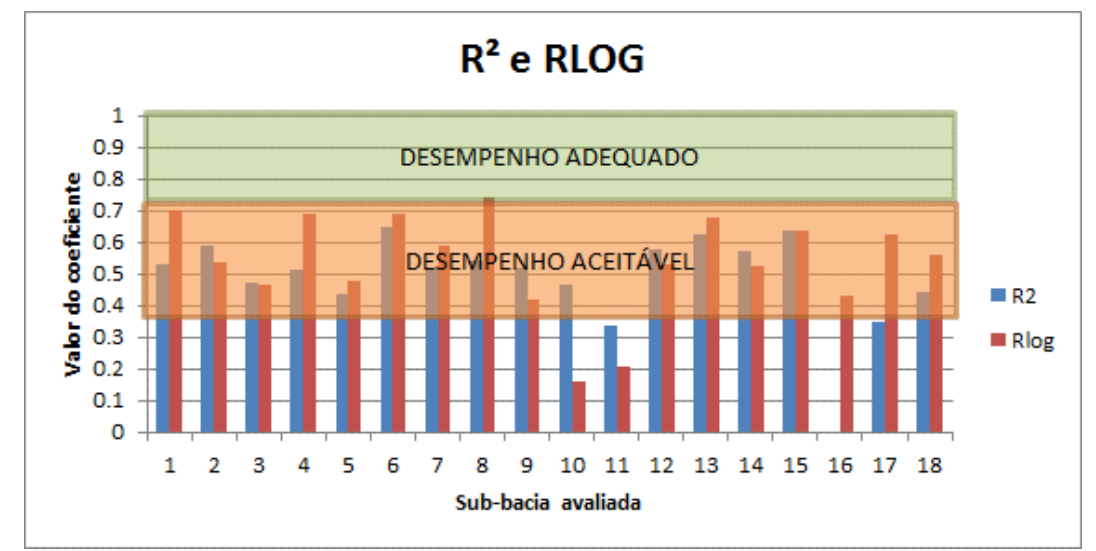

Figura 12 - Coeficientes de Eficiência Alcançados para a Bacia do Rio Doce

Avalia-se que também que os resultados obtidos possam ser otimizados e melhor calibrados, principalmente a partir da calibração automática existente no MGB-IPH não utilizada neste estudo. Uma melhor calibração proporcionaria melhores resultados futuros no que diz respeito às vazões e velocidade de escoamento nas minibacias, o que resultaria em estudos de deslocamento de ondas de cheia sobre as vazões de referência com maior acurácia.

Por fim, mesmo para um longo período de dados de 44 anos que dificulta a calibração do modelo, a curva de permanência obtida para o exutório da bacia (Figura 10 e Figura 11) denota o aceitável acoplamento entre as vazões observadas da bacia e as vazões estimadas pelo modelo chuvavazão utilizado. 


\section{5 - REFERÊNCIAS BIBLIOGRÁFICAS}

COLlischONN, W. ; AllASIA, D. G. ; SILVA, B. C. ; TUCCI, C. E. M. The MGB-IPH model for largescale rainfall-runoff modelling. Hydrological Sciences Journal, v. 52, p. 878-895, 2007

INSTITUTO BRASILEIRO DE GEOGRAFIA E ESTATÍSTICA. Mapa de Solos do Brasil. Acessado em 24/12/2014.<ftp://geoftp.ibge.gov.br/mapas_tematicos/mapas_murais/solos.pdf>

INSTITUTO BRASILEIRO DE GEOGRAFIA E ESTATÍSTICA. Mapa de Uso da Terral. 2010. Acessado em 24/12/2014.<ftp://geoftp.ibge.gov.br/mapas_tematicos/mapas_murais/uso_da_terra_ 2010.pdf>

INSTITUTO BRASILEIRO DE MEIO AMBIENTE E DOS RECURSOS NATURAIS RENOVÁVEIS. Laudo Técnico Preliminar - Impactos Ambientais Decorrentes do Desastre Envolvendo o Rompimento da Barragem de Fundão, em Mariana, Minas Gerais. Novembro de 2015.

MAPWINDOW, MapWindow GIS Open Source Project Ver 4.8.5. Idaho State University GSL. Disponível em: http://www.mapwindow.org/.

PASSOS, T.S.C.N. Barragem de Rejeito: Avaliação dos Parâmetros Geotécnicos de Rejeitos de Minério de Ferro Utilizando Ensaios de Campo - Um Estudo de Caso. 2009. [Trabalho de Conclusão de Curso] Curitiba (PR): Universidade Federal do Paraná.

SILVA, P. M. de O.; MELLO, C. R. de.; SILVA, A. M. da.; COELHO, G. Modelagem da Hidrógrafa de Cheia em uma Bacia Hidrográfica da Região Alto Rio Grande. Revista Brasileira de Engenharia Agrícola e Ambiental, v.12, n.3, mai.-jun., p.258-265, 2008.

SRTM, Shuttle Radar Topographic Mission. Consortium for Spatial Information CGIAR-CSI, http://srtm.csi.cgiar.org/

TUCCI, C. E. M. Modelos Hidrológicos. ABRH, 2.ed. Porto Alegre: Ed. UFRGS, 2005. 


\section{6 - ANEXOS}

\begin{tabular}{|c|c|c|c|c|c|c|c|c|c|c|c|c|c|c|c|}
\hline & & & & & & & & & & & & & & & \\
\hline $\begin{array}{c}\text { Sub-Bacia } 1 \\
\text { uge }\end{array}$ & & & & & & & & Sub-Bacia 4 & & & & & & & \\
\hline Past $R$ use & $\begin{array}{r}\text { Wm } \\
300.0\end{array}$ & $\begin{array}{r}b \\
0.15\end{array}$ & $\begin{array}{l}\text { Kabas } \\
0.90\end{array}$ & $\begin{array}{l}\text { Kint } \\
5.00\end{array}$ & $\begin{array}{r}\mathrm{XI} \\
0.60\end{array}$ & $\begin{array}{l}\text { CAP } \\
0,0\end{array}$ & Wc & use & $\mathrm{Wm}$ & b & Kbas & Kint & $\mathrm{XL}$ & CAP & Wc \\
\hline Past ${ }^{-n}$ & 400.0 & 0.15 & 0.90 & 5.00 & 0.60 & 0.00 & 0.10 & Past_R & 600.0 & 0.10 & 1.00 & 10.00 & 0.60 & 0.00 & 0.10 \\
\hline $\mathrm{Urb}-{ }^{2}$ & 300.0 & 0.10 & $\begin{array}{l}1.10 \\
1.00\end{array}$ & 6.00 & 0.60 & 0.00 & 0.10 & Past_P & 600.0 & 0.10 & 1.50 & 10.00 & 0.60 & 0.00 & 0.10 \\
\hline FlO $R$ & 400.0 & 0.15 & 0.90 & $\begin{array}{l}5.00 \\
5.00\end{array}$ & $\begin{array}{l}0.60 \\
0.60\end{array}$ & 0.00 & 0.10 & Urb & 800.0 & 0.10 & 0.50 & 10.00 & 0.60 & 0.00 & 0.10 \\
\hline F10_P & 500.0 & 0.15 & 1.00 & 6.00 & 0.60 & $\begin{array}{l}0.00 \\
0.00\end{array}$ & $\begin{array}{l}0.10 \\
0.10\end{array}$ & Flo_R & 700.0 & 0.10 & 2.00 & 10.00 & 0.60 & 0.00 & 0.10 \\
\hline $\mathrm{Lav}^{-} \mathrm{R}$ & 400.0 & 0.10 & 0.50 & 5.00 & 0.60 & 0.00 & 0.10 & $\begin{array}{l}\text { Flo-P } \\
\text { Lav } R\end{array}$ & 800.0 & 0.10 & 3.00 & 10.00 & 0.60 & 0.00 & 0.10 \\
\hline Lav_P & 500.0 & 0.10 & 0.60 & 6.00 & 0.60 & 0.00 & 0.10 & $\begin{array}{l}\text { Lav_R } \\
\text { Lav_P }\end{array}$ & $\begin{array}{l}400.0 \\
600.0\end{array}$ & $\begin{array}{l}0.10 \\
0.10\end{array}$ & $\begin{array}{l}1.80 \\
1.20\end{array}$ & $\begin{array}{l}10.00 \\
10.00\end{array}$ & $\begin{array}{l}0.60 \\
0.60\end{array}$ & $\begin{array}{l}0.00 \\
0.00\end{array}$ & 0.10 \\
\hline Aguà & 0.0 & 0.00 & 0.00 & 0.00 & 0.00 & 0.00 & 0.00 & Aguä & 0.0 & 0.00 & 0.00 & 0.00 & 0.00 & 0.00 & $\begin{array}{l}0.10 \\
0.00\end{array}$ \\
\hline cS & 10.00 & & & & & & & $\mathrm{cs}$ & 10.00 & & & & & & \\
\hline CI & 70.00 & & & & & & & CI & 70.00 & & & & & & \\
\hline CB & 1500.00 & & & & & & & CB & 2500.00 & & & & & & \\
\hline QB_M3/SKM2 & 0.0100 & & & & & & & QB_M3/SKM42 & 0.0100 & & & & & & \\
\hline Sub-Bacia 2 & & & & & & & & Sub-Bacia 5 & & & & & & & \\
\hline use & Wm & b & Kbas & Kint & $\mathrm{XI}$ & CAP & Wc & $\begin{array}{c}\text { che } \\
\text { uge }\end{array}$ & Wm & b & Kbas & Kint & XL & CAP & Wc \\
\hline Past_R & 790.0 & 0.10 & 0.30 & 5.00 & 0.60 & 0.00 & 0.10 & Past_R & 400.0 & 0.10 & 1.90 & 20.00 & 0.60 & 0.00 & 0.10 \\
\hline Past_P & 900.0 & 0.10 & 2.00 & 25.00 & 0.60 & 0.00 & 0.10 & Past_p & 550.0 & 0.10 & 2.90 & 15.00 & 0.60 & 0.00 & 0.10 \\
\hline Urb & 400.0 & 0.10 & 1.00 & 5.00 & 0.60 & 0.00 & 0.10 & $\mathrm{Urb}^{-}$ & 300.0 & 0.10 & 1.00 & 10.00 & 0.60 & 0.00 & 0.10 \\
\hline F10_R & 500.0 & 0.10 & 1.20 & 5.00 & 0.60 & 0.00 & 0.10 & - F10_R & 500.0 & 0.10 & 2.80 & 40.00 & 0.60 & 0.00 & 0.10 \\
\hline F10_p & 800.0 & 0.10 & 2.20 & 10.00 & 0.60 & 0.00 & 0.10 & F10_p & 700.0 & 0.10 & 3.90 & 30.00 & 0.60 & 0.00 & 0.10 \\
\hline Lav_ $R$ & 600.0 & 0.10 & 0.40 & 5.00 & 0.60 & 0.00 & 0.10 & Lav_R & 400.0 & 0.10 & 2.90 & 20.00 & 0.60 & 0.00 & 0.10 \\
\hline Lav_p $_{-}^{-P}$ & 500.0 & 0.10 & 1.50 & 10.00 & 0.60 & 0.00 & 0.10 & 0 Lav-p & 600.0 & 0.10 & 2.90 & 15.00 & 0.60 & 0.00 & 0.10 \\
\hline Aguāà & 0.0 & 0.00 & 0.00 & 0.00 & 0.00 & 0.00 & 0.00 & 0 Aguä & 0.0 & 0.00 & 0.00 & 0.00 & 0.00 & 0.00 & 0.00 \\
\hline cs & 10.00 & & & & & & & cs & 10.00 & & & & & & \\
\hline CI & 10.00 & & & & & & & CI & 70.00 & & & & & & \\
\hline CB & 1200.00 & & & & & & & $\mathrm{CB}$ & 2500.00 & & & & & & \\
\hline $\mathrm{QB}_{-} \mathrm{M} 3 / \mathrm{SKM} 2$ & 0.0100 & & & & & & & QB M3/SKM2 & 0.0100 & & & & & & \\
\hline $\begin{array}{c}\text { Sub-Bacia } 3 \\
\text { use }\end{array}$ & $\mathrm{Wm}_{\mathrm{m}}$ & b & Kbas & Kint & $\mathrm{xI}$ & CAP & พc & Sub-Bacia 6 & $\mathrm{Wm}$ & $b$ & Kbas & Kint & $\mathrm{XI}$ & CAP & Wc \\
\hline Past_R & 350.0 & 0.10 & 0.50 & 10.00 & 0.60 & 0.00 & 0.10 & Past_R use & 500.0 & 0.10 & 2.40 & 10.00 & 0.60 & 0.00 & 0.10 \\
\hline Past_P & 550.0 & 0.10 & 3.00 & 10.00 & 0.60 & 0.00 & 0.10 & Past_p & 600.0 & 0.10 & 3.90 & 10.00 & 0.60 & 0.00 & 0.10 \\
\hline Uxb & 150.0 & 0.10 & 0.50 & 10.00 & 0.60 & 0.00 & 0.10 & Uxb & 400.0 & 0.10 & 1.90 & 10.00 & 0.60 & 0.00 & 0.10 \\
\hline F10_R & 650.0 & 0.10 & 2.00 & 10.00 & 0.60 & 0.00 & 0.10 & Flo_R & 600.0 & 0.10 & 3.00 & 10.00 & 0.60 & 0.00 & 0.10 \\
\hline $\mathrm{F} 10_{-}^{-} \mathrm{P}$ & 900.0 & 0.10 & 4.00 & 10.00 & 0.60 & 0.00 & 0.10 & F10_p & 800.0 & 0.10 & 4.00 & 10.00 & 0.60 & 0.00 & 0.10 \\
\hline Lav_R & 350.0 & 0.10 & 0.70 & 10.00 & 0.60 & 0.00 & 0.10 & Lav_R $^{-1}$ & 500.0 & 0.10 & 0.90 & 10.00 & 0.60 & 0.00 & 0.10 \\
\hline Lav_p $^{-}$ & 550.0 & 0.10 & 3.00 & 10.00 & 0.60 & 0.00 & 0.10 & Lav_P $^{-1}$ & 600.0 & 0.10 & 2.40 & 10.00 & 0.60 & 0.00 & 0.10 \\
\hline Aguà & 0.0 & 0.00 & 0.00 & 0.00 & 0.00 & 0.00 & 0.00 & Aguà & 0.0 & 0.00 & 0.00 & 0.00 & 0.00 & 0.00 & 0.00 \\
\hline cs & 10.00 & & & & & & & $\mathrm{cs}$ & 10.00 & & & & & & \\
\hline CI & 70.00 & & & & & & & CI & 70.00 & & & & & & \\
\hline CB & 2700.00 & & & & & & & $\mathrm{CB}$ & 4500.00 & & & & & & \\
\hline QB_M3/SKM12 & 0.0100 & & & & & & & QB M3/SKML2 & 0.0100 & & & & & & \\
\hline Sub-Bacia ? & & & & & & & & Sub-Bacia 10 & & & & & & & \\
\hline use & $\mathrm{Wm}$ & b & Kbas & Kint & $\mathrm{xL}$ & CAP & พc & use & Wm & b & Kbas & Kint & $\mathrm{xL}$ & CAP & พc \\
\hline Past_R & 600.0 & 0.10 & 4.00 & 10.00 & 0.60 & 0.00 & $0.10=$ & Past_R & 600.0 & 0.10 & 1.00 & 10.00 & 0.60 & 0.00 & 0.10 \\
\hline Past_p & 800.0 & 0.10 & 4.00 & 10.00 & 0.60 & 0.00 & 0.10 & Past_p & 800.0 & 0.10 & 2.00 & 10.00 & 0.60 & 0.00 & 0.10 \\
\hline Urb & 800.0 & 0.10 & 4.00 & 10.00 & 0.60 & 0.00 & 0.10 & Urb & 800.0 & 0.10 & 1.00 & 10.00 & 0.60 & 0.00 & 0.10 \\
\hline Flo_R & 600.0 & 0.10 & 4.00 & 10.00 & 0.60 & 0.00 & $0.10=$ & Flo_R & 600.0 & 0.10 & 1.00 & 10.00 & 0.60 & 0.00 & 0.10 \\
\hline Flo_p & 800.0 & 0.10 & 4.00 & 10.00 & 0.60 & 0.00 & 0.10 & Flo_p & 800.0 & 0.10 & 2.00 & 10.00 & 0.60 & 0.00 & 0.10 \\
\hline $\mathrm{Lav}_{-}^{-} \mathrm{R}$ & 600.0 & 0.10 & 4.00 & 10.00 & 0.60 & 0.00 & 0.10 & Lav_R $^{-} R$ & 600.0 & 0.10 & 1.00 & 10.00 & 0.60 & 0.00 & 0.10 \\
\hline Lav_p $^{-1}$ & 800.0 & 0.10 & 4.00 & 10.00 & 0.60 & 0.00 & 0.10 & Lav_P $^{-} \mathrm{P}$ & 800.0 & 0.10 & 2.00 & 10.00 & 0.60 & 0.00 & 0.10 \\
\hline Aguā & 0.0 & 0.00 & 0.00 & 0.00 & 0.00 & 0.00 & 0.00 & Aguá- & 0.0 & 0.00 & 0.00 & 0.00 & 0.00 & 0.00 & 0.00 \\
\hline cs & 10.00 & & & & & & & cs & 10.00 & & & & & & \\
\hline CI & 70.00 & & & & & & & CI & 70.00 & & & & & & \\
\hline $\mathrm{CB}$ & 1500.00 & & & & & & & $\mathrm{CB}$ & 3000.00 & & & & & & \\
\hline QB_M3/SFM2 & 0.0100 & & & & & & & QB M3/SKM42 & 0.0100 & & & & & & \\
\hline Sub-Bacia 8 & & & & & & & & Sub-Bacia 11 & & & & & & & \\
\hline use & Wm & b & Kbas & Kint & $\mathrm{xL}$ & CAP & Wc & use & $\mathrm{Wm}$ & b & Kbas & Kint & $\mathrm{XL}$ & CAP & พc \\
\hline Past_R & 400.0 & 0.25 & 3.00 & 10.00 & 0.60 & 0.00 & $0.10=$ & Past_R & 500.0 & 0.10 & 0.50 & 10.00 & 0.60 & 0.00 & 0.10 \\
\hline Past_p & 500.0 & 0.25 & 5.00 & 5.00 & 0.60 & 0.00 & 0.10 & Past_p & 600.0 & 0.10 & 1.00 & 10.00 & 0.60 & 0.00 & 0.10 \\
\hline Urb & 500.0 & 0.25 & 3.00 & 10.00 & 0.60 & 0.00 & 0.10 & Urb & 500.0 & 0.10 & 0.50 & 10.00 & 0.60 & 0.00 & 0.10 \\
\hline F10_R & 500.0 & 0.25 & 3.00 & 10.00 & 0.60 & 0.00 & 0.10 & Flo_R & 600.0 & 0.10 & 1.00 & 10.00 & 0.60 & 0.00 & 0.10 \\
\hline F10_P & 650.0 & 0.25 & 4.00 & 10.00 & 0.60 & 0.00 & 0.10 & F10_p & 800.0 & 0.10 & 0.50 & 10.00 & 0.60 & 0.00 & 0.10 \\
\hline Lav_ $R$ & 500.0 & 0.25 & 3.50 & 10.00 & 0.60 & 0.00 & 0.10 & Lav_R & 300.0 & 0.10 & 0.50 & 10.00 & 0.60 & 0.00 & 0.10 \\
\hline Lav $_{-}{ }^{P}$ & 650.0 & 0.25 & 4.00 & 10.00 & 0.60 & 0.00 & 0.10 & Lav_p $^{-0}$ & 400.0 & 0.10 & 1.00 & 10.00 & 0.60 & 0.00 & 0.10 \\
\hline Aguă & $\begin{array}{r}0.0 \\
15.00\end{array}$ & 0.00 & 0.00 & 0.00 & 0.00 & 0.00 & 0.00 & Aguà & 0.0 & 0.00 & 0.00 & 0.00 & 0.00 & 0.00 & 0.00 \\
\hline CS & $\begin{array}{l}15.00 \\
70.00\end{array}$ & & & & & & & cs & 10.00 & & & & & & \\
\hline $\begin{array}{l}\mathrm{CI} \\
\mathrm{CB}\end{array}$ & $\begin{array}{r}70.00 \\
7000.00\end{array}$ & & & & & & & CI & 70.00 & & & & & & \\
\hline $\begin{array}{l}\text { CB } \\
Q^{B} \mathrm{M} 3 / \mathrm{SKM} 2\end{array}$ & 0.0100 & & & & & & & CB & 1500.00 & & & & & & \\
\hline QB_M3/SKM2 & 0.0100 & & & & & & & QB_M3/SKM2 & 0.0100 & & & & & & \\
\hline Sub-Bacia 9 & & & & & & & & Sub-Bacia 12 & & & & & & & \\
\hline use & Wm & b & Kbas & Kint & $\mathrm{XL}$ & CAP & พc & use & $\mathrm{Wm}$ & b & Kbas & Kint & $\mathrm{xL}$ & CAP & พc \\
\hline Past_R & 600.0 & 0.10 & 4.00 & 10.00 & 0.60 & 0.00 & $0.10_{\mathrm{P}}$ & Past_R & 600.0 & 0.20 & 2.00 & 10.00 & 0.60 & 0.00 & 0.10 \\
\hline Past_p & 800.0 & 0.10 & 4.00 & 10.00 & 0.60 & 0.00 & $0.10 \mathrm{P}$ & Past_P & 600.0 & 0.20 & 2.50 & 10.00 & 0.60 & 0.00 & 0.10 \\
\hline Uxb & 800.0 & 0.10 & 4.00 & 10.00 & 0.60 & 0.00 & $0.10 \mathrm{U}$ & Urb & 800.0 & 0.20 & 2.00 & 10.00 & 0.60 & 0.00 & 0.10 \\
\hline Flo_R & 600.0 & 0.10 & 4.00 & 10.00 & 0.60 & 0.00 & $0.10 \mathrm{~F}$ & Flo_R $R$ & 600.0 & 0.20 & 2.00 & 10.00 & 0.60 & 0.00 & 0.10 \\
\hline F10_P & 800.0 & 0.10 & 4.00 & 10.00 & 0.60 & 0.00 & $0.10 \mathrm{~F}$ & Flo_p & 700.0 & 0.20 & 3.00 & 10.00 & 0.60 & 0.00 & 0.10 \\
\hline Lav_R $^{-} R$ & 600.0 & 0.10 & 4.00 & 10.00 & 0.60 & 0.00 & $0.10 \mathrm{I}$ & $\mathrm{Lav}_{\mathrm{B}}^{-} \mathrm{R}$ & 600.0 & 0.20 & 2.00 & 10.00 & 0.60 & 0.00 & 0.10 \\
\hline Lav_P $_{-}^{-}$ & 800.0 & 0.10 & 4.00 & 10.00 & 0.60 & 0.00 & $0.10 \mathrm{I}$ & Lav_p & 800.0 & 0.20 & 3.00 & 10.00 & 0.60 & 0.00 & 0.10 \\
\hline Aguāa & 0.0 & 0.00 & 0.00 & 0.00 & 0.00 & 0.00 & $0.00 \mathrm{~A}$ & Aguă & 0.0 & 0.00 & 0.00 & 0.00 & 0.00 & 0.00 & 0.00 \\
\hline cs & 10.00 & & & & & & & CS & 17.00 & & & & & & \\
\hline CI & 70.00 & & & & & & & CI & 70.00 & & & & & & \\
\hline CB & 1500.00 & & & & & & & $C B$ & 1500.00 & & & & & & \\
\hline QB_M3/SKM2 & 0.0100 & & & & & & & QB M3/SKM2 & 0.0100 & & & & & & \\
\hline
\end{tabular}




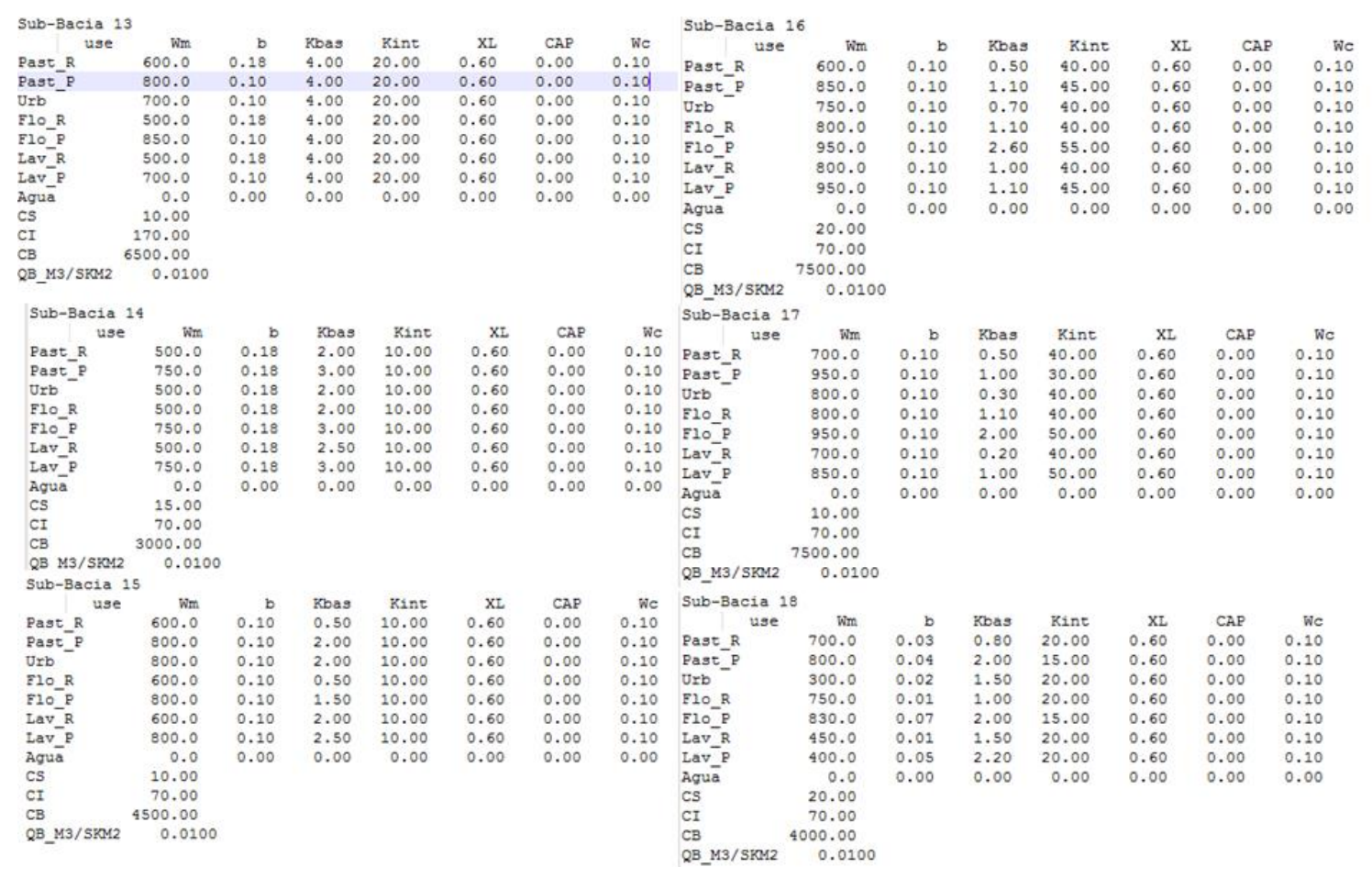

